NIHON REOROJĪ GAKKAISHI Vol. 24, No. 1, 11 14 (1996)

(C)1996 The Society of Rheology, Japan

\title{
Numerical Solution of Flow of a Bingham Fluid on a Rotating Disk ${ }^{\dagger}$
}

\author{
Fan $\mathrm{CHUN}^{* 1}$, Chen YaOsong ${ }^{* 2}$ \\ ${ }^{* 1}$ Institute of Mechanics, Chinese Academy of Sciences, Beijing 100080, China \\ ${ }^{* 2}$ Department of Mechanics, Peking University, Beijing 100871, China
}

(Received: November 25, 1994)

Equations describing the flow of a film of a Bingham fluid on a rotating disk were derived. The equations considered evaporation of a solvent involved in the fluid and the resulting changes in rheological parameters. From those equations, the film thickness distribution in the radial direction was numerically evaluated with the difference method. Two kinds of the thickness distribution were found. (1) For the case of initial yield stress $\tau_{0}=0.01$ and rheological parameter $\alpha \geq 0.1$, the thickness $h$ increased with increasing radial coordinate $r$. This result agreed with the experimental result for magnetic disks used in electronic computers. (2) For the cases of $\alpha=0$ and $\tau_{0}=0.01 ; 0.1 ; 0.2, h$ decreased with increasing $r$. This result agreed with the experimental result obtained by Jenekhe and Schuldt.

Key Words: Bingham Fluid/Rotating Disk/Numerical Solution

\section{INTRODUCTION}

In many industries it is necessary to coat a thin film on a surface, and spin-coating is a simple, widely used technique. The first description of the flow of a viscous fluid on a rotating disk was given by Emslie ${ }^{1)}$, the same problem for a power law fluid by Acrivos $^{2)}$, for a Bingham fluid by Matsumoto ${ }^{3)}$, and for a Casson fluid by Chan Man Fong ${ }^{4)}$. In these studies the time-dependent viscosity was not considered. That is, the studies neglected an effect of evaporation of solvents involved in the fluids. The concentration $C$ of the solute increases due to evaporation of the solvent, and both viscosity and yield stress increase rapidly with increasing $C$. In this paper we shall consider this effect and describe the flow of a Bingham fluid, using Chan Man Fong's order-of-magnitude comparison ${ }^{4)}$.

\section{EQUATION OF MOTION AND CONSTITUTIVE EQUATION}

We choose a cylindrical polar coordinate system $(\bar{r}, \bar{\theta}, \bar{z})$ with the origin at the centre of the disk. For a film of a Bingham fluid placed on the disk, we assume the physical components of the velocity to be

$$
v_{r}=\bar{u}, v_{\theta}=\bar{r} \Omega, v_{z}=\bar{w}
$$

\footnotetext{
†Supported by National Natural Science Foundation of China.
}

where $\Omega$ is the constant angular speed of the disk.

We define the dimensionless variables as follows:

$$
\begin{aligned}
& r=\bar{r} / a ; z=\bar{z} / h_{0} ; t=\left(h_{0} / a\right) \Omega \bar{t} ; \\
& u=\bar{u} / h_{0} \Omega ; w=a \bar{w} / h_{0}^{2} \Omega ; \\
& P=\bar{P} / \rho h_{0}^{2} \Omega^{2} ; T_{i j}=\bar{T}_{i j} / \rho h_{0}^{2} \Omega^{2}
\end{aligned}
$$

where $a$ is the radius of the disk, $h_{0}$ is the initial thickness of the film, $\bar{t}$ is the time, $\bar{P}$ is the pressure, $\rho$ is the density, $\bar{T}_{i j}$ is the deviatoric stress tensor.

All dimensionless quantities are assumed to be of the same order-of-magnitude.

We assume the rotating motion to be symmetry.

The equations of motion for the Bingham fluid are

$$
\begin{aligned}
\frac{\partial u}{\partial t}+ & u \frac{\partial u}{\partial r}+w \frac{\partial u}{\partial z}-\frac{a^{2}}{h_{0}^{2}} r \\
& =-\frac{\partial P}{\partial r}+\frac{\partial T_{r r}}{\partial r}+\frac{a}{h_{0}} \frac{\partial T_{r z}}{\partial z}+\frac{T_{r r}-T_{\theta \theta}}{r} \\
2 \frac{a}{h_{0}} u & =\frac{1}{r} \frac{\partial}{\partial r}\left(r T_{r \theta}\right)+\frac{a}{h} \frac{\partial T_{z \theta}}{\partial z} \\
\frac{\partial w}{\partial t}+ & u \frac{\partial w}{\partial r}+w \frac{\partial w}{\partial z} \\
& =-\frac{a^{2}}{h_{0}^{2}} \frac{\partial P}{\partial z}+\frac{a}{h_{0}} \frac{\partial}{\partial r}\left(r T_{r z}\right)+\frac{a^{2}}{h_{0}^{2}} \frac{\partial T_{z z}}{\partial z}
\end{aligned}
$$

The equation of continuity is

$$
\frac{1}{r} \frac{\partial(r u)}{\partial r}+\frac{\partial w}{\partial z}=0
$$


The boundary conditions are

(a) no slip on the disk

$$
z=0, u=w=0
$$

(b) on the free surface, the shear stress must vanish

$$
z=h\left(=\bar{h} / h_{0}\right), T_{i j}=0 \quad(i \neq j)
$$

where $h$ is the thickness of the film and is a function of $\bar{t}$ and $\bar{r}$.

We define the dimensionless quantities for the Bingham fluid as follows:

$$
\begin{aligned}
& A_{i j}=\bar{A}_{i j} / \Omega ; K(C)=\bar{K}(C) / \rho h_{0}^{2} \Omega ; \\
& \tau_{y}(C)=\bar{\tau}_{y}(C) / \rho h_{0}^{2} \Omega^{2}
\end{aligned}
$$

where $C$ is the concentration of the solute, $\bar{A}_{i j}$ is the Rivlin-Ericksen tensor of first order, and $\bar{K}(C)$ and $\bar{\tau}_{y}(C)$ are the $C$-dependent plastic viscosity and yield stress. The constitutive equation of the Bingham fluid is written in terms of those quantities as

$$
\begin{gathered}
T_{i j}=\left\{K(C)+\tau_{y}(C) /\left|A_{s t} A_{t s} / 2\right|^{1 / 2}\right\} A_{i j}, \\
\quad \text { for } T_{s t} T_{t s} / 2 \geq \tau_{y}^{2}, \\
A_{i j}=0 \text { for } T_{s t} T_{t s} / 2<\tau_{y}{ }^{2}
\end{gathered}
$$

According to the assumption of rotational symmetry, we find from eqs 1 and 2 that $A_{\mathrm{ij}}$ is given by

$$
A=\left[\begin{array}{ccc}
\frac{2 h_{0}}{a} \frac{\partial u}{\partial r} & 0 & \frac{h_{0}^{2}}{a^{2}} \frac{\partial w}{\partial r}+\frac{\partial u}{\partial z} \\
0 & \frac{2 h_{0}}{a} \frac{u}{r} & 0 \\
\frac{h_{0}^{2}}{a^{2}} \frac{\partial w}{\partial r}+\frac{\partial u}{\partial z} & 0 & \frac{2 h_{0}}{a} \frac{\partial w}{\partial z}
\end{array}\right]
$$

The solute concentration $C$ changes on evaporation of a solvent involved in the Bingham fluid. The evaporation rate in turn depends on the relative velocity between the fluid surface and air. The relative velocity for the fluid on the rotating disk is given by $r \Omega$. Thus we may assume that the rate of the change in $C$ varies linearly with $r \Omega$. This assumption neglects the evaporation at a quiescent state and becomes invalid at low $\Omega$. However, at large $\Omega$ of our interest, the evaporation is mostly induced by the rapid rotation of the disk and the assumption becomes reasonable. The evaporation rate is also dependent on $C$. The rate decreases with increasing $C$ and we assume, for simplicity, that the rate varies linearly with $C_{\mathrm{S}}-C$, with $C_{\mathrm{S}}$ being the concentration at solidification. Under these assumptions, we may describe the change in $C$ by

$$
\partial C / \partial t=\bar{\alpha} r \Omega\left(C_{s}-C\right)
$$

where $\bar{\alpha}$ is a constant. From this equation, we obtain

$$
C=C_{s}-\left(C_{s}-C_{0}\right) \exp (-\bar{\alpha} r \Omega \bar{t})
$$

with $C_{0}$ being the initial concentration.

\section{DIFFERENTIAL EQUATION FOR THE FILM THICK NESS}

We assume $h_{0} / a \ll 1$. From (8) we compare the first term of $A_{\text {rz }}$ with the second one

$$
\frac{h_{0}^{2}}{a^{2}} \frac{\partial w}{\partial r} / \frac{\partial u}{\partial z} \approx\left(h_{0} / a\right)^{2} \ll 1
$$

thus,

$$
A_{r z}=\partial u / \partial z
$$

Using the same order-of-magnitude comparison, we may deduce that $A_{r r} / A_{r z}=\left(h_{0} / a\right) \ll 1, A_{\theta \theta}$ and $A_{z z}$ are of the same order of magnitude as $A_{r r}$. So $A_{r r}, A_{\theta \theta}$ and $A_{z z}$ can be neglected. From (7) it can be seen that the comparison between $T_{r z}$ and $T_{r r}, T_{\theta \theta}, T_{z z}$ is as the same as the comparison between $A_{r z}$ and $A_{r r}, A_{\theta \theta}, A_{z z}$. So $T_{r r}, T_{\theta \theta}$ and $T_{z z}$ can be neglected. The constitutive equation (7) is then simplified to

$$
\begin{aligned}
& T_{r z}=\tau_{y}(C) \operatorname{sgn}\left(\frac{\partial u}{\partial z}\right)+K(C) \frac{\partial u}{\partial z}, \text { for }\left|T_{r z}\right| \geqslant \tau_{y}, \\
& \partial u / \partial z=0, \quad \text { for }\left|T_{r z}\right|<\tau_{y}
\end{aligned}
$$

where $\operatorname{sgn}(\partial u / \partial z)$ takes the value +1 for a positive argument and -1 for a negative argument.

The equation of motion (3) can then be simplified to

$$
\left(a / h_{0}\right) r=\partial T_{r z} / \partial z
$$

From (11) we see that the motion due to the centrifugal force is determined in term of the balance between the centrifugal force and viscous force. We can also see that the motion is a shear flow in the $r$-direction superimposed on a rotational rigid body motion in the $\theta$-direction.

The Bingham fluid becomes solid, that is, both the plastic viscosity $K(C)$ and the yield stress $\tau_{y}(C)$ tend to $\infty$ as $C \rightarrow C_{s}$.

Thus we assume

$$
K(C)=B /\left(C_{s}-C\right)=B \exp (\bar{\alpha} r \Omega \bar{t}) /\left(C_{s}-C_{0}\right)
$$

where $B$ is a constant. $K(C)$ is rewritten as

$$
K(C)=K_{0} \exp (\alpha r t)
$$

where $K_{0}=K\left(C_{0}\right)=B /\left(C_{\mathrm{s}}-C_{0}\right), K_{0}$ is the initial plastic viscosity, and $\alpha=\bar{\alpha} a / h_{0}$ is a constant.

In the same way, we may write $\tau_{\mathrm{y}}(C)$ as

$$
\tau_{\mathrm{y}}(C)=\tau_{0} \exp (\beta r t)
$$

where $\tau_{0}$ is the initial yield stress, and $\beta$ is a constant.

The solution of (10), (11), (12a), and (12b) subject to the boundary condition (5) and (6) is

$$
\begin{array}{ll}
u=R_{e} \exp (-\alpha r t) r\left[\xi^{2}-(\xi-z)^{2}\right] / 2, & \xi \geqslant z \geqslant 0 ; \\
u=R_{e} \exp (-\alpha r t) r \xi^{2} / 2, & h \geqslant z \geqslant \xi
\end{array}
$$

where $R_{e}=\rho a h_{0} \Omega / K_{0}$ is the Reynolds number. $z=\xi$ is the interface between fluid-like behaviour and solid-like behaviour:

$$
\xi=h-\tau_{y} / R_{e} r
$$

Since $\xi$ cannot be negative, so the flow region is restricted to

$r \geqslant \tau_{y} / R_{e} h$

To determine the film thickness $h$ at any time $t$, we integrate (4) across the film at any fixed point $r$, that is, we integrate it over $z$, from $z=0$ to $z=h$. Using the kine- 
matic condition at the interface and free surface, we obtain:

$$
\begin{aligned}
\frac{\partial h}{\partial t} & +\frac{1}{r}\left[\int_{0}^{\xi} u(r, z) d z+\int_{\xi}^{h} u(r) d z\right] \\
& +\frac{\partial}{\partial r} \int_{0}^{\xi} u(r, z) d z+\frac{\partial}{\partial r} \int_{\xi}^{h}(r) d z=0
\end{aligned}
$$

Substituting (13) into (16), after some manipulation, we obtain the differential equation of film thickness as follow:

$$
\begin{aligned}
& \frac{e^{\alpha r t}}{R_{e}} \frac{\partial h}{\partial t}+r h\left(h-\frac{\tau_{y}}{R_{e}} \frac{1}{r}\right) \frac{\partial h}{\partial r}+\frac{1}{6}\left(h-\frac{\tau_{y}}{R_{e}} \frac{1}{r}\right) \\
& {\left[4 h^{2}+h \frac{\tau_{y}}{R_{e}} \frac{1}{r}+\left(\frac{\tau_{y}}{R_{e}} \frac{1}{r}\right)^{2}\right]-\frac{r t}{3}\left(h-\frac{\tau_{y}}{R_{e}} \frac{1}{r}\right)} \\
& \left\{\alpha h^{2}+\left(\frac{3}{2} \beta-\frac{1}{2} \alpha\right)\left[h \frac{\tau_{y}}{R_{e}} \frac{1}{r}+\left(\frac{\tau_{y}}{R_{e}} \frac{1}{r}\right)^{2}\right]\right\}=0
\end{aligned}
$$

\section{NUMERICAL SOLUTION AND DISCUSSION}

The partial differential equation (17) is solved numerically using the difference method. The parameter values used are the radius of disk $a=20 \mathrm{~cm}$, the initial thickness $h_{0}$ $=20 \mu \mathrm{m}$, the initial plastic vicosity $K=1.2 \mathrm{~g} / \mathrm{cms}$, the density

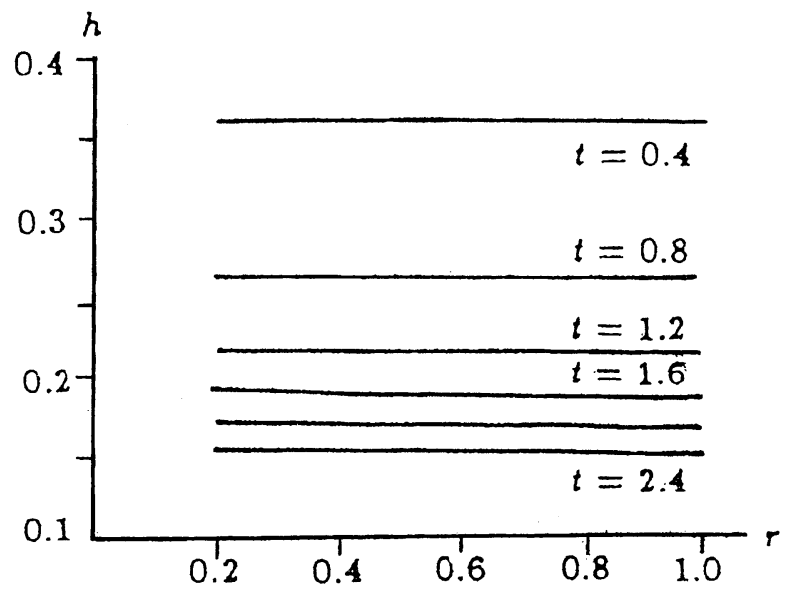

Fig. 1 The thickness $h$ as a function of $r$ for various values of $t$ for the case of $\tau_{0}=0.01, \alpha=0, \beta=0$.

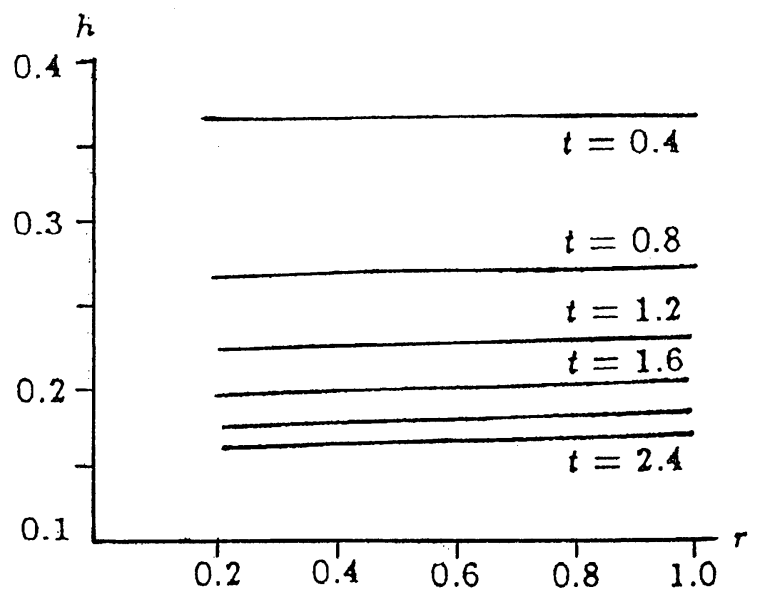

Fig. 2 The thickness $h$ as a function of $r$ for various values of $t$ for the case of $\tau_{0}=0.01, \alpha=0.2, \beta=0.4$. $\rho=1.2 \mathrm{~g} / \mathrm{cm}$, the angular speed of disk $\Omega=3000 \mathrm{rpm}$ and the initial thickness distribution $h(0, r)=1$ for the cases of $\tau_{0}=$ $0.01,0.1,0.2$ and $\alpha, \beta$, being $0.1,0.2$, and 0.4 respectively.

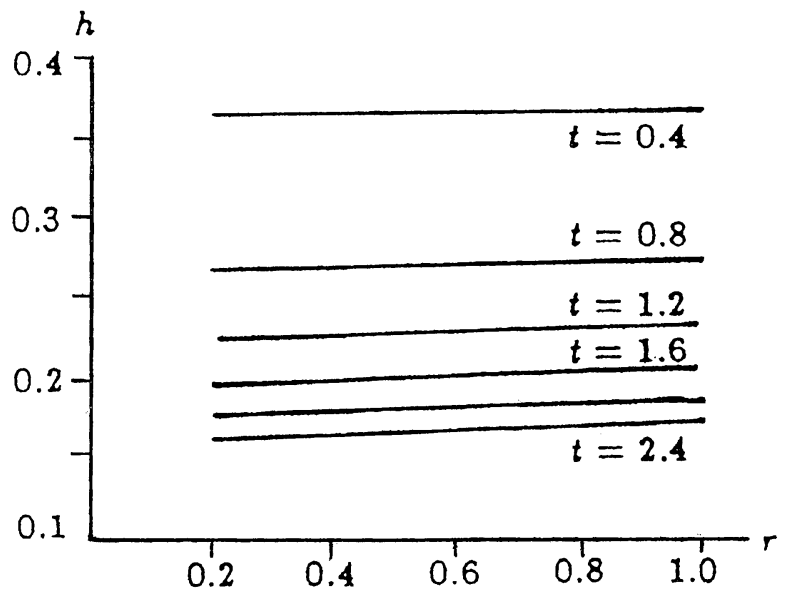

Fig. 3 The thickness $h$ as a function of $r$ for various values of $t$ for the case of $\tau_{0}=0.01, \alpha=0.4, \beta=0.1$.

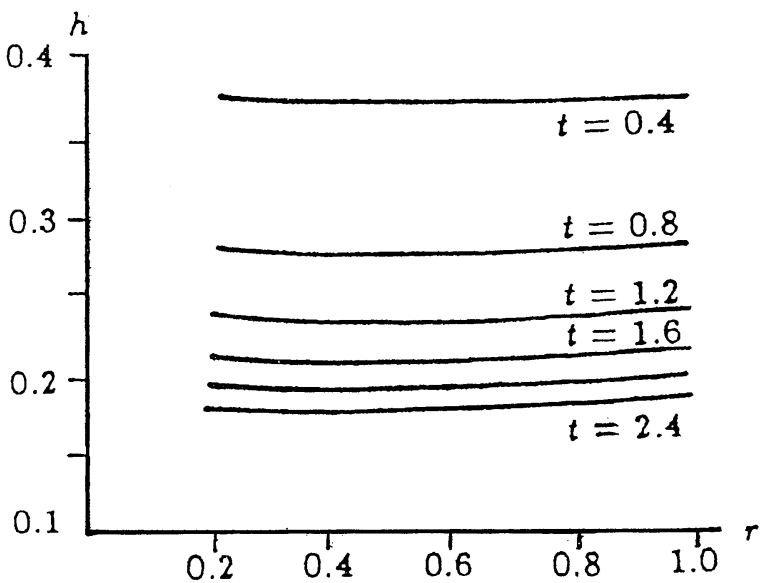

Fig. 4 The thickness $h$ as a function of $r$ for various values of $t$ for the case of $\tau_{0}=0.1, \alpha=0.6, \beta=0.3$.

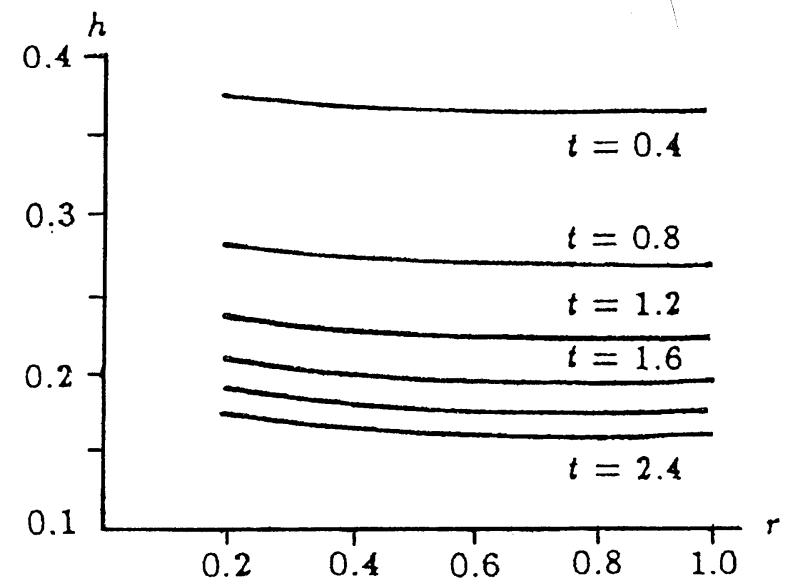

Fig. 5 The thickness $h$ as a function of $r$ for various values of $t$ for the case of $\tau_{0}=0.1, \alpha=0, \beta=0.1$. 


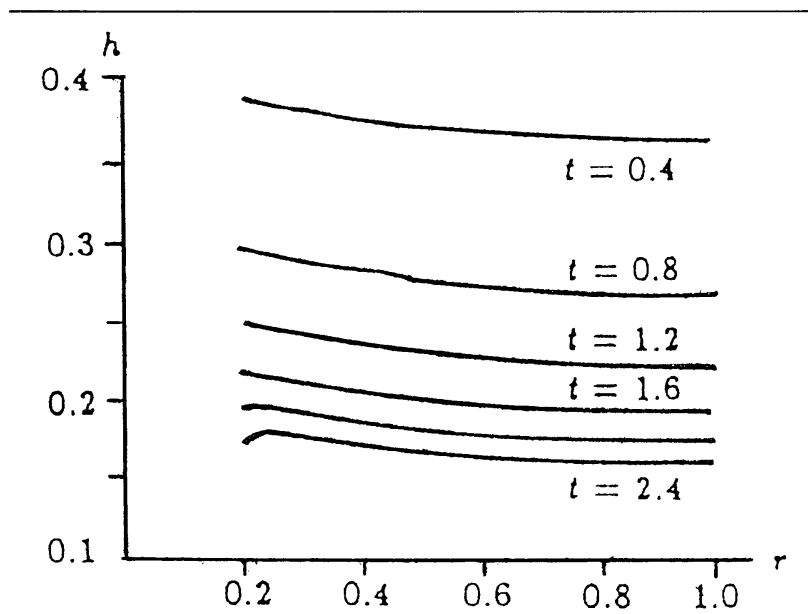

Fig. 6 The thickness $h$ as a function of $r$ for various values of $t$ for the case of $\tau_{0}=0.2, \alpha=0, \beta=0$.

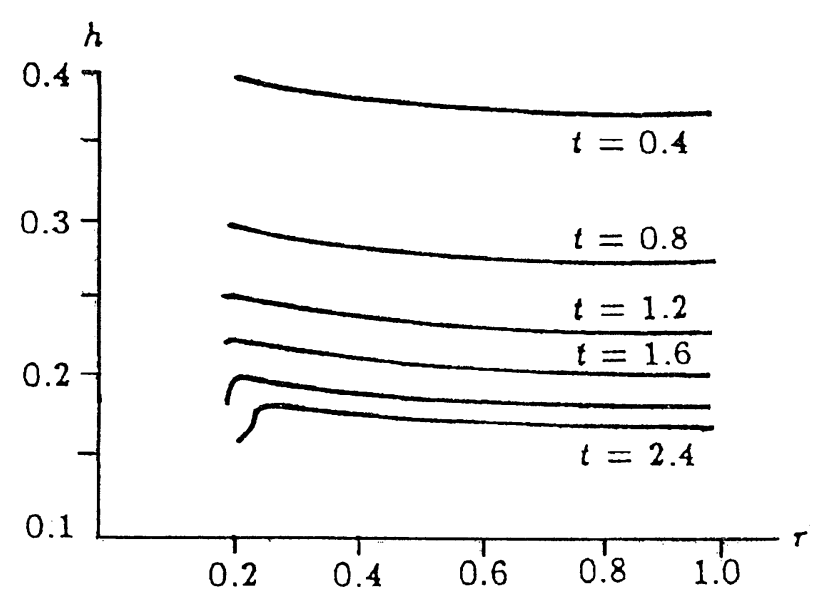

Fig. 7 The thickness $h$ as a function of $r$ for various values of $t$ for the case of $\tau_{0}=0.2, \alpha=0, \beta=0.3$.

Figs. 1 7 show the film thickness $h$ as a function of $r$ for various values of $t$ for the cases of $\tau_{0}=0.01,0.1$, and 0.2 . From Figs. 1 7, we can see two kinds of the thickness distribution.
(1) From Figs. 2 and 3 for the cases of $\tau_{0}=0.1, \alpha=0.6$ and $\beta=0.3$, we can see that the thickness $h$ increases with increasing $r$. This result agrees with the experimental result for the magnetic disk used in electronic computers. The inclination of the thickness inceases with increasing $\alpha$.

(2) From Figs. 1, 5, and 6 for the cases of $\alpha=0$ and $\tau_{0}=$ $0.01,0.1$, and 0.2 , we can see that the thickness $h$ decreases with increasing $r$. This result agrees with the experimental result of Jenekhe and Schuldt ${ }^{5)}$. The inclination of the thickness decreases with increasing $\tau_{0}$.

(3) Figs. 6 and 7 are for the case of $\tau_{0}=0.2$, according to Eq. (15), $\beta$ is restricted to $\beta<0.2$.

In conclusion, the $\alpha$ makes the thickness $h$ increase with increasing $r$. The $\tau_{0}$ is just the opposite; it makes the thickness $h$ decrease with increasing $r$. The effect of $\beta$ on the thickness distribution is small.

\section{ACKNOWLEDGEMENT}

Authors wish to thank Professors C. F. Chan Man Fong of Sherbrooke University, Canada, Bian Yin-gui and Zhu $\mathrm{Ru}-z e n g$ of Institute of Mechanics, Chinese Academy of Scineces and the referee for their helpful comments and suggestions.

\section{REFERENCES}

1) Emslie AG, Bonner FT, Peck LG, J Appl Phys, 29, 858 (1958).

2) Acrivos A, Shan MJ, Petersen EE, J Appl Phys, 31, 963 (1960).

3) Shiro Matsumoto, Yoichi Takashima, et al, $I \& E C$ Fundamentals, 21, 198 (1982).

4) CF Chan Man Fong, Cai Fushi, Xu Yuanze, ACTA Mechanica Sinica, 3, 107 (1987).

5) Jenekhe SA, Schuldt SB, Chen Eng Commun, 33, 135 (1985). 